\title{
Die Dynamik in der kulturellen Veränderung und Institutionalisierung
}

\author{
Von LAURI HONKO
}

Es ist kaum richtig, die Anwendung des Terminus 'Dynamik' auf die ersten Phasen der Entwicklung einer religiösen Bewegung zu begrenzen. Daß eine solche Abgrenzung mitunter in religionswissenschaftlichen Betrachtungen durchscheint, kann jedoch nicht auf ein einfaches semantisches Versäumnis zurückgeführt werden (selbstverständlich haben auch institutionalisierte Formen religiöser Aktivität oder religiöse Institutionen ihre Dynamik), sondern auf ein gewisses Idealbild des Institutionalisierungsprozesses selbst. Liest man jene 21 Kriterien durch, mit deren Hilfe L. Pope den Übergang vom Typus Sekte zum Typus Kirche beschreibt ${ }^{1}$ oder blättert man in Th. O'Deas ,,fünf Dilemmas" oder ,fünf Paradoxen“" in der Institutionalisierung $^{2}$, merkt man leicht, wie der Typus Sekte oder die Situation 'noch nicht institutionalisierte Bewegung' mit Charakteristika geladen sind wie Spontanität, Enthusiasmus, Charisma, Anschaulichkeit, egalitäre Teilnahme, moralische Gemeinschaft, Zufriedenstellung aller Bedürfnisse, Expressivität, eine Mehrzahl von Gottesdienstformen, kollektive Administration, volkstümliche Kirchenlieder, Gewicht religiöser Häuslichkeit etc. Der Typus Kirche gestaltet sich als etwas Stabiles und Starres, Bürokratisches und Hierarchisches, Kompromißbereites und halb Säkularisiertes, Wohlorganisiertes und Wohlkontrolliertes, aber wenig Inspirierendes und Macht-, aber nicht Menschenorientiertes. Diese idealtypische, zugespitzte Polarität kann eventuell wenigstens einige Unterstützung in empirischen Untersuchungen finden, falls man innerhalb einiger ausgewählter christlicher Gesellschaftsmilieus verbleibt. Sie repräsentiert somit zunächst ein kulturgebundenes Modell, das sich am besten zur Untersuchung

\footnotetext{
${ }^{1}$ Cf. Pope $122 \mathrm{ff}$.

${ }^{2}$ Cf. O'Dea, 1961, $30 \mathrm{ff}$ und 1966, $90 \mathrm{ff}$.
} 
jener Gemeinschaft eignet, innerhalb der sich das Modell selbst entwickelt hat. Eine größere Übertragbarkeit auf andere Kulturen besitzt es kaum. Diese Begrenzung garantiert jedoch nicht die Objektivität des Modells, nicht einmal für die abendländische industrielle und postindustrielle Gesellschaftsform. Der Schöpfer und Meister der Idealtypanalyse, M. Weber, hat von so vielen Epigonen eine so einhellige Unterstützung bekommen, daß er - falls er noch lebte - vielleicht eine Theorie des symptomatischen Verhaltens der Religionssoziologie vor einer tiefgehenden kulturellen und sozialen Veränderung aufstellen könnte.

Das von uns gezeichnete Bild des Prozesses der Institutionalisierung hätte sich nie lediglich aufgrund einiger empirischer Untersuchungen etablieren können. Das Resultat empirischer Forschungen sieht oft ganz anders aus als die Hypothesen, die den Ausgangspunkt bildeten. Oft kommt es zu alternativen Auslegungsmöglichkeiten und ganz anderen Fragestellungen als vom Forscher anfangs angenommen. Bestimmte soziologische Untersuchungen, die sich vorgenommen haben, eine Hypothese auf Punkt und Komma zu verifizieren und nichts anderes zu tun, wirken wie Maßarbeit und sind alles andere als überzeugend für den Kulturforscher.

Wenn also unser Bild vom Institutionalisierungsprozeß nicht als direktes Resultat empirischer Untersuchungen aufgefaßt werden kann, dürfte die Frage angebracht sein, zu welchen Perspektivfehlern es führen kann, wenn das Bild trotz allem schief ist und die Wirklichkeit einseitig wiedergibt. Und bezüglich des einseitigen Bildes kann man fragen, warum es sich denn so konstituiert: ist es vielleicht doch mehr eine Funktion unserer Lebensoder Kultursituation denn der Forschungstradition?

Spontane, mitunter kurzlebige Kulte und neugeschaffene, oft kontroverse Sekten sind hinsichtlich ihres Formats erfahrungsgemäß geeigneter für empirische Untersuchungen als große Religionsgemeinschaften und Kirchen. Dadurch ist eventuell unsere Auffassung vom Institutionalisierungsprozeß in gewissem Ausmaß gefärbt worden. Der emotionelle Einsatz, der zu jeder Forschung gehört, nicht zuletzt zur humanistischen und sozialwissenschaftlichen, ist im Fall der Religionsforschung den Klein- oder Teilreligionen zugute gekommen. Mikrostudien und Fallstudien sind immer allgemeiner geworden aufkosten von Makrostudien und vergleichenden Untersuchungen auf breiterer Basis. Das hat jedoch nicht dazu geführt, daß sich das Hauptinteresse der abendländischen Religionsforschung auf die außerkirchlichen Sekten und Kulte gerichtet hätte. Ein bedeutender 
Teil der Primärforschung ist im Rahmen einer größeren Gemeinschaft vom Typ Kirche durchgeführt worden. Die Frage nach dem Format ist auf eine ähnliche Weise gelöst worden: als Studienobjekt wurden innerkirchliche Protestbewegungen gewählt, interessante charismatische Persönlichkeiten, die Dynamik der religiösen Erweckung u.dgl.m., d.h. eindeutig abgegrenzte religiöse Phänomene und Teilkulturen. Makrostudien hat es nicht sonderlich viele gegeben, es sind vor allem einzelne religionsgeographische oder religionssoziologische Querschnitte statistischen Charakters.

Trotz des offenbaren Interesses der Religionswissenschaft für Kleinreligionen, Teilreligionen und Erweckungsbewegungen, die recht oft einen Protest gegen eine größere und institutionalisiertere Religionsform enthalten, haben wir ein ziemlich großes quellenkritisches Problem gerade angesichts des Studiums vom Anfangsstadium der Kleinreligionen. Wieviele Religionsforscher haben letzten Endes das Aufkommen einer religiösen Bewegung beweisen und die Persönlichkeit des charismatischen Führers selbst dokumentieren können, die Bedeutung der Anhängerschaft und die eventuellen Konflikte angesichts des organisatorischen und Machtproblems, das dann zur einen oder anderen Form der Institutionalisierung führt? Die Anzahl primärer Beobachtungen dieser Art ist sicher viel geringer als die Menge der sekundären Quellen, die zwar das Aufkommen der Bewegung, das Charisma des Führers usw. beschreiben, aber verfaßt und umredigiert sind von einem Anhänger der Bewegung. Daß der Forscher seine Analyse auf in-grouporientierter Literatur aufbauen muß, ist ein Sachverhalt, der sicher seine Spuren hinterläßt: ziemlich viel Information, die sicher akzeptierte Tradition darstellt, nicht aber durch andere Quellen kontrolliert werden kann, wird in die wissenschaftliche Vorstellung hineinfiltriert. Hinzu kommt oft eine mehr oder minder aktive Identifikation des Forschers mit dem Wertsystem der Bewegung, etwas also, was mitunter als eine Form teilnehmender Beobachtung charakterisiert werden kann, was aber gleichzeitig zumindest in bestimmten Fällen den Forscher an Schlußfolgerungen hindert, die Wertsystem und Zielsetzung jener religiösen Bewegung in Frage stellen würden.

Wenn es also in unserem Idealbild von der Ausgangsphase der Institutionalisierung Perspektivfehler gibt, kann dies auf die Situation des Forschers und den Charakter des Quellenmaterials zurückgeführt werden. Eine gewisse Überbetonung des 'positiven' Elements in der Entstehungsgeschichte einer Bewegung ist zu erwarten, sie beruht sowohl auf dem 
Charakter der religiösen Tradition als auch auf der Einstellung des Forschers. Es scheint, als könne u. a. die religionssoziologische Theoriebildung hier durchdrungen werden von der grundlegenden Bedeutung und Heiligkeit der Ursprungsgeschichten und Mythen. Der Heroskult kann nicht allein durch negative Quellenkritik abgeschafft werden, vor allem wenn jegliche Zusatzinformation fehlt, die das Bild ausgleichen und jenes Vakuum mit Inhalt ausfüllen könnte, das bei der Quellenkritik stets zu hinterbleiben tendiert.

Warum nimmt sich eine vollendete Institutionalisierung in diesem Zusammenhang wie etwas Drohendes aus? Das ist eine tendenziöse Frage, berechtigt nur durch die vorhandene Tendenz im oben skizzierten polaren Idealtyp. Hier kann es sich nicht um Entwicklungsphasen außerhalb der Reichweite der empirischen Forschung handeln. Eher im Gegenteil: wenn man von bestimmten neuen und eventuell kurzlebigen Religionsbildungen absieht, kann man sagen, daß wir es rein quantitativ fast ausschließlich mit fest institutionalisierten Religionsformen zu tun haben, wenn wir alle religiösen Aktivitäten in unserer Umwelt studieren. Auch die meisten Sekten weisen einen Grad an Institutionalisierung auf, der auch in dem Fall nicht wegerklärt werden kann, wo die Bewegung Dogmen aufrechterhält, die direkt gegen die Bürokratie und einen hierarchischen Anschluß an andere Gemeinschaften kämpfen. Es gibt also gute Möglichkeiten zur empirischen Forschung über die Funktionsweise der religiösen Institutionen. Hier sollen weder Vorstellungen noch Vorurteile dominieren. Verwunderlich ist, daß man in dieser Situation in gewissem Umfang geneigt ist, ein Interesse am Studium der Dynamik der Kirchen, religiösen Gemeinschaften und diverser Teilinstitutionen auf eine nicht-theologische, vorzugsweise religionssoziologische Weise zu verneinen. Die Tatsache, daß diese Institutionen die Möglichkeiten haben, eigene Forscher mit besserem Zugang zu bestimmtem Quellenmaterial zu rekrutieren, dürfte hier eine ganz unbedeutende Rolle spielen. Auch das Bewahren einer nicht zu soziologischen Fachidentität ist von untergeordneter Bedeutung. Ein etwas wichtigerer Faktor dürfte darin liegen, daß die Methodologie der Religionswissenschaft nicht über das vollständige Rüstzeug verfügt, alle von diesem Gesichtspunkt relevanten Probleme aufzugreifen, für welche die Sozialwissenschaften Analysemethoden entwickelt haben. Während man einräumt, daß eine engere Zusamm enarbeit mit bestimmten Sozialwissenschaften sehr aktuell wäre für die Religionswissenschaft, kann man gleichzeitig darauf hinweisen, daß die historischen, 
phänomenologischen und anthropologischen Richtungen der Religionswissenschaft einiges zu bieten haben bei der Erstellung einer interdisziplinären Forschungsstrategie, z. B. wenn man die Entwicklung der religiösen Institutionen auch im Makrosystem untersuchen will. Hat der Religionsforscher kein Interesse für die Funktionsweise der großen machthabenden und machtausübenden religiösen Institutionen, für deren innere und äußere Dynamik und Entwicklungsprofile, kann man leicht sagen, daß sein Blick abgewandt ist von Welt und Gesellschaft.

Wenn sich die Religionswissenschaft eine menschenzentrierte Froschperspektive zugelegt hat, so ist das mehr auf der Grundlage von Individuen und Gruppen geschehen, die eine ungewöhnlich starke religiöse Aktivität aufweisen und einen gewissen Protestgeist gegen die teilweise säkularisierte Kirche. Auf das abendländische Bild vom homo religiosus hat das relativ passive, religiös fast indifferente Mitglied einer Kirche sehr wenig eingewirkt. Seine quantitative Bedeutung ist jedoch unbestreitbar groß und kann nicht übersehen werden. Das passive, stille Mitglied ist weiterhin ein religionssoziologisches Problem, das z. B. in den Untersuchungen über die Säkularisierung sichtlich nicht gelöst wurde. In diesen Untersuchungen wird oft ein früherer, a priori mehr religiöser Abschnitt als Ausgangspunkt genommen, und das Resultat kann ziemlich uninteressant sein, insbesondere wenn neue Formen der Religiosität außerhalb der Analyse bleiben und die Faktoren, die die Kontinuität der religiösen Zugehörigkeit tragen, so verborgen bleiben wie vorher.

Symptomatischerweise hat die Religionswissenschaft eine ziemlich fortgeschrittene Sektologie entwickelt. Denkt man an B. Wilsons Aufbau einer deckenden Taxonomie der Sektenformen ${ }^{3}$ oder an J. Yingers Typologie der religiösen Organisationen ${ }^{4}$, merkt man, daß wir bereits über eine beachtliche Anzahl idealtypischer Kategorien verfügen, in denen die empirisch betrachteten religiösen Grippierungen mehr oder minder reibungslos untergebracht werden können. Hinsichtlich Wilsons kann festgestellt werden, daß er den Begriff Sekte praktisch bis zum äußersten dehnen mußte und daß die Wirklichkeit selten reine Repräsentanten seiner Sektentypen aufweist; es gibt Bewegungen, die sich nicht leicht in seine Typologie einordnen lassen. Yinger hatte teilweise mit demselben Problem zu tun, hat

${ }^{3}$ Cf. Wilson $7 \mathrm{ff}$.

${ }^{4}$ Cf. Yinger $251 \mathrm{ff}$. 
aber die herkömmliche Dimension Kirche-Sekte besser bewahrt. Sein Versuch der Konfrontation nichtchristlicher Religionen mit christlichen Religionsformen ist interessant, nicht zuletzt deshalb, weil sich herausstellt, daß dieselben Kriterien in unterschiedlicher kultureller Umgebung nicht auf dieselbe Weise wirken.

Sind Typologien dieser Art eine Lösung für die Untersuchung des Prozesses der Institutionalisierung? Eine strategische Schwäche scheint darin zu liegen, daß jeder Typ ein Augenblicksbild einer religiösen Bewegung oder deren idealisierter Form wiedergibt. Die Untersuchung des Institutionalisierungsprozesses ist vor allem das Studium einer Entwicklung. Es muß die verschiedenen Phasen aufweisen können, durch welche die Entwicklung einer Bewegung gegangen ist, und jene Indikatoren finden, die den Übergang von einer Phase zur anderen am besten markieren. Dies ist etwas anderes, als eine Anzahl religiöser Bewegungen in typologische Schubladen zu stecken und sie da zu lassen. Es gilt, den Weg der religiösen Gruppe im kulturellen wie im sozialen Kontext zu verfolgen. Diese Kontexte werden mindestens ebenso wichtig wie der eigene Traditionsvorrat der religiösen Gruppe. Typologische Kategorien obengenannter Art scheinen nicht besonders effektiv zu sein, wenn man ein Entwicklungsprofil für eine religiöse Gruppe skizzieren muß. Der in typologischer Hinsicht distinktive Zug kann eine längere Zeit bestehen, während der eine markante Entwicklung vor sich geht. Man benötigt daher einen größeren Vorrat nicht von Organisationstypen, sondern von Organisationskriterien und anderen Entwicklungsindikatoren, um ein Entwicklungsprofil zeichnen zu können.

Wenn ich vom Entwicklungsprofil einer Bewegung spreche, bedeutet das, daß man zunächst eine empirische Anknüpfung an eine Bewegung und deren kulturelles und soziales Milieu hat. Der komparative Aspekt ist jedoch keineswegs ausgeschlossen. Mit der Zeit werden andere Bewegungen im selben Milieu eingeschaltet oder man geht über zu interkulturellen Vergleichen in Zeit und Raum. Eine historisch-deskriptive Darstellung dessen, wie es mit einer religiösen Bewegung zuging, wird nie zu einer Entwicklungsanalyse, obwohl erstere eine nützliche Unterlage für letztere sein kann. Das potentielle Abstraktionsniveau einer Entwicklungsanalyse variiert mitunter gerade im Hinblick auf die komparative Aufstellung (Zeit, Raum, Anzahl Kulturen etc.). Ich glaube, hier kann die Religionswissenschaft einiges von sog. interkulturellen Untersuchungen pro- 
fitieren, wie die Anthropologen sie nach dem Zweiten Weltkrieg entwickelt haben. Ich verweise auf die Untersuchungen von $\mathbb{R}$. Carneiro und das von R. Naroll und R. Cohen redigierte methodologische Handbuch ${ }^{5}$. Sie haben mit Entwicklungsprofilen experimentiert, die mit Hilfe ausgewählter Indikatoren prinzipiell das Profil der gesamten Kultur im Hinblick auf deren Entwicklungstrend beschreiben.

Das eigentliche Ziel der interkulturellen anthropologischen Forschung liegt darin, so viele Kulturen wie möglich miteinander zu vergleichen. Dieses Ziel kann natürlich auch vom Religionsforscher akzeptiert werden. Doch sind das relativ hohe Abstraktionsniveau und das summarische Verfahren der interkulturellen Anthropologie nicht die einzige Möglichkeit. Ich glaube, man könnte den Vergleichsaspekt etwas einschränken und im Prinzip den Gesamtüberblick über das Kulturmilieu und die Gesellschaft beibehalten. Man kann sich recht spannende Untersuchungen über die Institutionalisierung einer religiösen Bewegung vorstellen, wo man ihren Entwicklungstrend und ihre Entwicklungsmöglichkeiten in einem breiteren kulturellen und sozialen Kontext sieht, wo auch andere als rein religiöse Aktivitäten der Menschen eingeschaltet werden mit dem Gedanken an eventuelle Korrelationen, die auch in anderen Zusammenhängen und Milieus entdeckt werden können. Der ganzheitliche Aspekt ist also kein Selbstzweck, sondern ein Weg zu gewissen nichtreligiösen kulturellen und sozialen Faktoren, die sich wiederholt als bedeutungsvoll erweisen, wenn man den Institutionalisierungsprozess vom Standpunkt seiner Entwicklung betrachtet.

Wir sind uns alle der metatheoretischen und praktischen Ungleichheiten bewußt, die zwischen den Verfahrensweisen des Kulturforschers, z.B. des Religionshistorikers, und des Sozialforschers, z.B. des Soziologen, bestehen. Das Studium der Institutionalisierung ist eine der wichtigsten Domänen der Sozialforscher gewesen. Doch wissen wir Kulturforscher nur zu gut, wie leicht der Soziologe an wichtigen Veränderungen im kulturellen Traditionsvorrat vorbeigehen und wie hilflos er sein kann, wenn er sie auf die eine oder andere Weise dokumentieren soll. Meiner Auffassung nach sind die kulturellen Veränderungen genauso wichtig wie die sozialen oder die ökonomischen Veränderungen, wenn man erklären will, weshalb der Prozeß der Institutionalisierung gerade das Profil hat, das

${ }^{5}$ Cf. Carneiro, 1970, 834 ff. Cf. ib., 1973, 89 ff. 
er hat. Diese ungleichen Veränderungen brauchen durchaus nicht im selben Tempo zu geschehen und haben nicht dieselbe Ausdehnung oder dasselbe Durchsetzungsvermögen. Was wir Institutionalisierung einer religiösen Bewegung nennen, ist nicht ausschließlich der Übergang einer sozialen Gruppe zu festeren und regulierteren Aktionsformen. Sie beinhaltet auch eine kulturelle Veränderung, eine neue Akzentuierung herkömmlicher Vorstellungen und Verhaltensmuster, das Verwerfen bestimmter Elemente in der religiösen Tradition, das Einführen von neuen Elementen aus dem latenten Traditionsvorrat und all das, um den Veränderungen in der Gruppenorganisation, im Autoritäts- und Machtverhältnis der Gruppe Leben und Bedeutung sowie Legitimation zu geben. Wie diese Traditionsveränderung sich eigentlich gestaltet, das hat der Kultur- und Religionsforscher zu klären, nicht der Soziologe.

Eine Zusammenfassung des Gesagten ergibt die folgenden Thesen:

1. Unsere Auffassung von der Institutionalisierung wird stark dominiert durch einen polaren Idealtyp (Kult od. Sekte-Kirche), gebunden an das abendländische industrielle Kulturmilieu. Auch wenn ein polarer Idealtyp rein theoretisch nicht der empirischen Wirklichkeit zu entsprechen braucht, kann er in bestimmten Fällen - sklavisch angewandt - zu einer einseitigen Orientierung in der Forschung führen. Man kann dann u. a. eine gewisse Überbewertung eines positiv eingeschätzten Elements in der Entstehungsgeschichte einer Bewegung registrieren sowie ein geringes Interesse für vergleichende Studien über die innere und äußere Dynamik der großen religiösen Institutionen.

2. Die Sektentypologien sind nicht die optimale Lösung als methodisches Instrument für die Untersuchungen über den Institutionalisierungsprozeß.

3. Die Institutionaliserung ist als Prozeß und Entwicklung nicht vielseitig genug untersucht worden. Es ist daher angebracht, eine Forschungsstrategie zur Anwendung zu bringen, wo religiöse und nichtreligiöse Entwicklungsindikatoren nebeneinander benutzt werden. Ein Entwicklungsprofil der Institutionalisierung wird nicht nur mit Hilfe von Organisationskriterien skizziert, sondern auch durch Kriterien, die die kulturelle oder Traditionsveränderung kennzeichnen und nicht direkt parallel verlaufen müssen zu den sozialen, ökonomischen usw. Veränderungen, mit ihnen aber in Verbindung stehen. 


\section{Literaturverzeichnis}

Carneiro, R., 1970, Scale Analysis, Evolutionary Sequences, and the Rating of Cultures. Handbook 1970.

- 1973, The Four Faces of Evolution. Handbook 1973.

Handbook, 1970, A Handbook of Method in Cultural Anthropology. Ed. by R. Naroll \& R. Cohen. New York \& London.

- 1973, Handbook of Social and Cultural Anthropology. Ed. by J. Honigmann. Chicago.

O'Dea, T., 1961, Five Dilemmas in the Institutionalization of Religion. Journal for the Scientific Study of Religion 1.

- 1966, The Sociology of Religion. Englewood Cliffs.

Pope, L., 1942, Millhands and Preachers. New Haven.

Wilson, B., 1970, Religious Sects. London.

Yinger, J., 1970, The Scientific Study of Religion. New York. 